\title{
RE: Consumer-based technology for distribution of surgical videos for objective evaluation
}

\author{
Daniel A. Hashimoto $\cdot$ Ernest D. Gomez • \\ Noel N. Williams $\cdot$ Rajesh Aggarwal
}

Received: 13 August 2012/Accepted: 10 October 2012/Published online: 12 December 2012

(C) Springer Science+Business Media New York 2012

We commend the authors of "Consumer-based technology for distribution of surgical videos for global evaluation" for utilizing easily accessible equipment as a means to distribute videos for evaluation of technical skill [1]. Given the ubiquity of mobile devices and the cumbersome nature of handling DVD multimedia, video distribution through mobile-enabled, web-based services certainly improves access to videos for assessment, feedback, quality control, and medical record keeping.

Facilitating access to surgical videos raises three key concerns based on data security and privacy. First, even when patient or surgeon identities are not apparent from video images, potentially identifying information, such as time and location of the operation, can be bundled with the video files in the form of metadata. Second, standards of security and privacy may be inconsistent among parties sending and receiving data. Finally, the results of skill evaluation are highly sensitive information given their potential implications for surgeon accreditation and malpractice. Thus, it is worth considering whether surgeons should be given the ability to opt out of sharing their procedure videos with third parties.

Quality of surgical practice is essential to patient safety, yet current methods of assessing surgical quality often focus on outcome measures, which, although effective, provide an incomplete picture of surgical quality and performance. Comparing surgical performance to elite athletics, consider that although the final score of a basketball game may reflect the winner, only a breakdown of each quarter and player can give a complete picture of how the game was won. Similarly, process measures assessed through means such as objective evaluation of video may be a valuable resource to highlight areas of good performance and identify deficiencies to correct.

The authors effectively demonstrate how advances in information technology will aid the surgical community as we strive to develop practical, meaningful methods for assessing surgical skill. However, we must continue to protect patient and surgeon privacy rights as skill assessment enters the 21 st century.

Disclosures Mr. Hashimoto, Mr. Gomez, and Drs. Williams and Aggarwal have no conflict of interest or financial ties to disclose.

\section{References}

1. Gonzalez R, Martinez J, Lo Menzo E, Iglesias A, Ro C (2012) Consumer-based technology for distribution of surgical videos for objective evaluation. Surg Endosc 26:2179-2182
D. A. Hashimoto - E. D. Gomez - N. N. Williams · R. Aggarwal Perelman School of Medicine, University of Pennsylvania, Philadelphia, PA, USA

\section{A. Hashimoto $(\bowtie) \cdot R$. Aggarwal}

Department of Biosurgery and Surgical Technology, St. Mary's Hospital, Imperial College London, 10th Floor Queen Elizabeth the Queen Mother Building, Praed Street, London W2 1NY, UK

e-mail: dhash@mail.med.upenn.edu 